\title{
CONTENT-BASED FILTERING FOR MUSIC RECOMMENDATION BASED ON UBIQUITOUS COMPUTING
}

\author{
Jong-Hun Kim', Un-Gu, Kang ${ }^{2}$, and Jung-Hyun Lee' \\ ${ }^{1}$ Department of Computer Science \& Engineering Inha University \\ Yonghyun-dong, Nam-gu, Incheon, Korea \\ jhkim@hci,inha.ac.kr, jhlee@inha.ac.kr \\ ${ }^{2}$ Department of Information Technology Gachon University of Medicine and Science \\ Yeonsu-dong, Yeonsu-ku, Incheon, Korea \\ ugkang@gachon.ac.kr
}

\begin{abstract}
In music search and recommendation methods used in the present time, a general filtering method that obtains a result by inquiring music information and recommends a music list using users' profiles is used. However, this filtering method presents a certain difficulty to obtain users' information according to their circumstances because it only considers users' static information, such as personal information. In order to solve this problem, this paper defines a type of context information used in music recommendations and develops a new filtering method based on statistics by applying it to a content-based filtering method. In addition, a recommendation system using a content-based filtering method that was implemented by a ubiquitous computing technology was used to support service mobility and distribution processes. Based on the results of the performance evaluation of the system used in this study, it significantly increases not only the satisfaction for the music selection, but also the quality of services.
\end{abstract}

Key words: Content-based Filtering, Ubiquitous Computing, OSGi

\section{INTRODUCTION}

The amount of multimedia data that is processed by users has exponentially increased since the development and popularization of the 
Internet. An increase in multimedia data presents difficulties in searching information within a user's desired time frame, which currently exceeds the limitation of data processing time for an individual user.

In order to solve these problems, a music search system that only provides the results of queries by rank using music information in a web environment has largely been used in the past and at the present time. Although a music search system has the advantage of being easily implemented, it may produce unwanted worthless information due to the exclusion of a user's interests and becomes a reason for a decrease in user satisfaction. In recent years, a recommendation system has been actively studied that predicts and recommends only information requested by a user. Various filtering methods are used in this recommendation system in order to estimate a user's preferences. For instance, a recommendation system using filtering methods have been used in some search engines such as Yahoo, Amazon online bookstore, and CDNow Internet shopping mall and have been well received by the user.

In this recommendation system, the similarity between the content of an item and user information was measured to recommend information desired by the user, and a content-based filtering method that based the rank on this measurement was also used. However, the recommendation of multimedia data is still limited [1] and is not highly reliable due to filtering only being based on static information. In particular, with a music recommendation system in the present web service environment, it is difficult to exactly recommend music that is desired by users because real-time context information like weather significantly affects a user's music selection.

Thus, this paper attempted to design a content-based music recommendation system (CBMRS) that is able to recommend music according to a user's interests and conditions by applying context information to a content-based filtering method. In order to apply context information to the CBMRS, data regarded as a factor in music selection was configured as context information and was based on ontology. In addition, data obtained using various sensors and an Radio Frequency Identification (RFID) Tag based on Open Service Gateway Initiative (OSGi) could be recognized as exact context information through an ontology database and inference engine. The recognized context information in this process could be used in a content-based filtering process based on statistics and also applied to provide a recommended list that was well received by the user. The system used in this study consisted of three large sections; a Context Manager section, Service Manager section, and Music Recommendation Manager section. The usefulness and results of this recommendation system satisfies a large number of persons in the results of the performance evaluation of the system used in this study. 


\section{CONTENT-BASED FILTERING USING CONTEXT INFORMATION}

This system defines contexts to recommend music by considering surrounding contexts and user information and configures a music list using a content-based filtering method. The content-based filtering used in a recommend process first configures a list by searching the music that corresponds to user preferences and user information from a Music Content Information Database (MCIDB) and builds an initial profile using this list.

The initial profile can be updated using the music title selected by users and the context information recognized in an OSGi environment in a music service. This profile is statistically analyzed and recommends a music list that corresponds to the context information from the MCIDB when a user inquires about a music service.

\subsection{Configuration and Definition of Context Information}

The definition of contexts created by Brown, defined information for a users' location or surroundings [2]. Brown's definition is an accurate method to develop application services, used to configure and determine proper context for a music recommendation service in this system.

This system determines the following factors which affect music selection: user sex, age, temperature, and weather before the configuration and determination of context.

The configuration of context information for the CBMRS consists of user information (sex, age, pulsation), weather, and outdoor temperature. In addition, user location information in the home is configured as context information. This allows a music recommendation service to employ certain applications regardless of the user's location in the home.

Table 1 presents the definition of context information as different spaces, such as class 2 for sex, class 5 for age, class 4 for pulsation, class 4 for temperature, class 7 for weather, and class 6 for location information. In particular, a pulsation below 40 or above 181 is defined as a dangerous condition to generate an event because the average pulsation of an adult is about $65 \sim 120$. In addition, the service area is limited to homes, and the users' location is limited to the Balcony, Bathroom, Guestroom, Kitchen, and Living-room.

This system was implemented the ubiquitous network based on an OSGi framework in order to acquire automatic sensing datas. User information, temperature, and location information can be input from sensors based on OSGi framwork. User sex, age, and location information can be traced using 
an RFID Tag which is attached to a user's watch, and pulsation information can be obtained from a pulse sensor which is attached to a user's watch through real-time Zigbee communication. However, although weather information is predefined as ontology, its data can be established as a database retrieved from the Internet.

Table 1. Configuration and Definition of Context Information

\begin{tabular}{|c|c|c|c|c|c|c|c|c|}
\hline Sex & \multicolumn{2}{|c|}{ Age } & \multicolumn{2}{|c|}{ Pulsation } & \multirow{2}{*}{$\begin{array}{c}\text { Weather } \\
\text { class }\end{array}$} & \multicolumn{2}{|c|}{ Temperature } & Location \\
\hline class & num. & class & num. & class & & $\mathrm{F}^{0}$ & class & class \\
\hline \multirow{3}{*}{$\begin{array}{l}\text { MA- } \\
\text { LE }\end{array}$} & $\begin{array}{l}0 \sim \\
7\end{array}$ & Infant & $\begin{array}{l}0 \sim \\
40\end{array}$ & Danger & \multirow{5}{*}{$\begin{array}{c}\text { Clear } \\
\text { Sunny } \\
\text { Cloudy } \\
\text { Shower } \\
\text { Rain } \\
\text { Snow } \\
\text { Storm }\end{array}$} & $\begin{array}{l}-4 \sim \\
30.2\end{array}$ & Cold & \multirow{5}{*}{$\begin{array}{c}\text { Balcony } \\
\text { Bathroom } \\
\text { Bedroom } \\
\text { Guestroom } \\
\text { Kitchen } \\
\text { Livingroom }\end{array}$} \\
\hline & $\begin{array}{l}8- \\
11 \\
\end{array}$ & Child & $\begin{array}{l}41 \sim \\
65 \\
\end{array}$ & Low & & $\begin{array}{l}32 \sim \\
68 \\
\end{array}$ & Cool & \\
\hline & $\begin{array}{l}12 \sim \\
17\end{array}$ & $\begin{array}{l}\text { Young } \\
\text { Adult }\end{array}$ & $\begin{array}{l}66 \sim \\
120\end{array}$ & Normal & & $\begin{array}{l}69.8 \sim \\
86\end{array}$ & Warm & \\
\hline \multirow{2}{*}{$\begin{array}{l}\text { FEM- } \\
\text { ALE }\end{array}$} & $\begin{array}{l}18 \sim \\
61\end{array}$ & Adult & $\begin{array}{l}121 \sim \\
180\end{array}$ & High & & $87.8 \sim$ & Hot & \\
\hline & $62 \sim$ & $\begin{array}{c}\text { Old } \\
\text { Adult }\end{array}$ & $181 \sim$ & Danger & & & & \\
\hline
\end{tabular}

The context of the CBMRS based on the context information used in this study is defined as Web Ontology Language (OWL) that is used on a Semantic Web in order to configure and express exact contexts and various relationships. Fig. 1 presents the hierarchy of OWL classes.

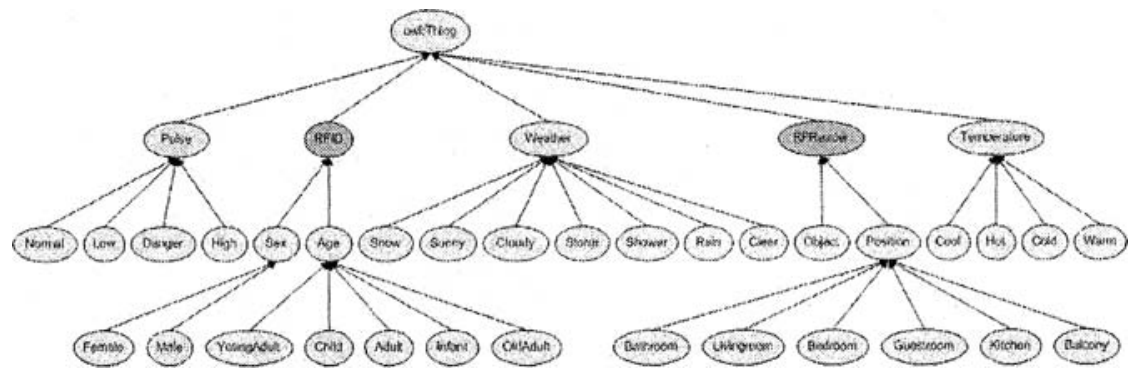

Fig.1. Ontology for Context in Content-based Music Recommendation System

\subsection{Content-based Filtering Based on Statistical Method}

At the present time, the filtering methods used in recommendation system are rule-based filtering, learning agent, content-based filtering, and collaborative filtering methods.

A rule-based filtering method identifies the preference of users and provides recommendations by connecting items to the characteristics of that 
item. The rule used in this method is predefined by experts. This method is effective with an item that has a high unit price and complicated characteristics. A content-based filtering method keeps the information related to items and recommends these items to the user who inputs a keyword, which is related to the property of the information. This method has been largely used in the field of information search [3]. A collaborative filtering method establishes a database according to the item preference of users and searches neighbors that have similar preferences when a new customer appears. In addition, the item that a neighbor is interested in is recommended to the customer under the assumption that the customer will be interested in the item [4].

This system remembers and creates a profile based on a selected item and context information when users are faced with specific context. In addition, when a user requires a recommended service, this system recommends a similar item to the user's former selection based on this profile. Thus, among previously mentioned filtering methods, a content-based filtering method can be used to configure a recommendation list.

To establish a Music Content Information Database (MCIDB), an automatic establishing method in web documents and user input methods were used. In an automatic establishing method, web documents can be extracted by a web robot agent. In addition, a database can be built using the analysis of morphology.

Table 2 shows the recorded value of the 'Winter Rain' in the MCIDB in which the key word was obtained by analyzing the song title using a morphological analysis.

Table 2. Example of a Music Content Information Database

\begin{tabular}{|c|c|}
\hline Music Information & Extracted Nouns \\
\hline Title & Winter Rain \\
\hline Singer & Earl Klugh \\
\hline Genre & Pop \\
\hline Age & Young Adult \\
\hline Weather & Rain \\
\hline Temperature & Cold \\
\hline Keyword & Winter, Rain \\
\hline
\end{tabular}

The earlier profile was configured as a title selected by the user according to the weather, temperature, and recommended ages from a music content information database. This means that context data, such as weather, temperature, and age profiles, could be obtained. Furthermore, a type of query profile is an additional profile configured using a music list which is selected from specific context. The query profile used in this profile becomes a selected music list according to the specific context of a user. For 
example, the profile can be automatically configured with the selected music list when context is presented as snow-cold-young_adult-normal. A profile can be produced according to a user's selected item for each context data set.

The recommendation method used in this system is as follows:

First, the frequency of words reappearing can be calculated using a morphological analysis method for a profile that corresponds to context information when a user requires a recommended service. For instance, if the context information is assumed to be snow-cold-young_adult-normal when a user requires a recommended service, the frequency of words reappearing can be calculated by analyzing the morphology of snow, cold, and young adult profiles. The frequency of a specific word reappearing can be expressed as $P\left(W_{1}\right)$ represented in Eq. (1).

$$
P\left(W_{i}\right)=\text { Freq }_{\max }\left(W_{i}\right) / \sum_{i=1}^{N} \text { Freq }_{\max }\left(W_{i}\right) \times 100, \quad 1 \leq i \leq N
$$

where $W_{1}$ is the $i$ th word in a specific profile, $F r e q_{\max }\left(W_{1}\right)$ is the total appearance number of $W$ in the profile, and $N$ is the total word in the profile except for the duplicated word.

Second, this method searches for the word that appears most frequently with each profile and song title, which coincides with the key word in the MCIDB and configures a list based on the results of these searches. Third, it combines these results into a single list excluding duplicate song titles. Fourth, it configures the priority of the list. In this process, the morphology of the query profile (snow-cold-young_adult-normal profile) that corresponds to the context information is analyzed and the word that appears with the highest frequency is extracted. In addition, a new list is configured according to the priority of the music, which coincides with the extracted key word from the query profile in the previously configured list. Moreover, music selected by users can be recorded in an inquiry profile, context data profiles and that be used as an accurate music list according to the repetition in selections.

\section{SYSTEM DESIGN AND IMPLEMENTATION}

This chapter designed and implemented the CBMRS that was able to recommend proper music by estimating context information in a Java-based OSGi framework using the context definition and filtering method proposed in Chapter 2.

The system proposed in this study developed an OSGi gateway using the Knopflerfish 1.3.3, an open architecture source project which implemented a service framework. The OSGi is a type of industrial standard proposed by an OSGi organization in order to establish a standard connection method for 
Internet devices, such as household information devices and security systems. It is JES-based gateway software, which is an open architecture Java embedded server able to provide high quality multimedia services with a high security level regardless of platform application software. In particular, it is an open architecture network technology that can support various network techniques, such as Bluetooth, Home Audio/Video Interoperability (HAVi), Home Phoneline Networking Alliance (PNA), Home Radio Frequency (RF), Universal Serial Bus (USB), Video Electronics Standards Association (VESA), and other networks [5].

Fig. 2 presents the diagram of the overall system. The CBMRS designed in this paper analyzed and suggested various data transferred from context recognition sensors and established it as information to recommend proper music through a filtering process for user profiles and MCIDB. In order to perform this process, the CBMRS consisted of a Context Manager, Service Manager, and Music Recommendation Manager.

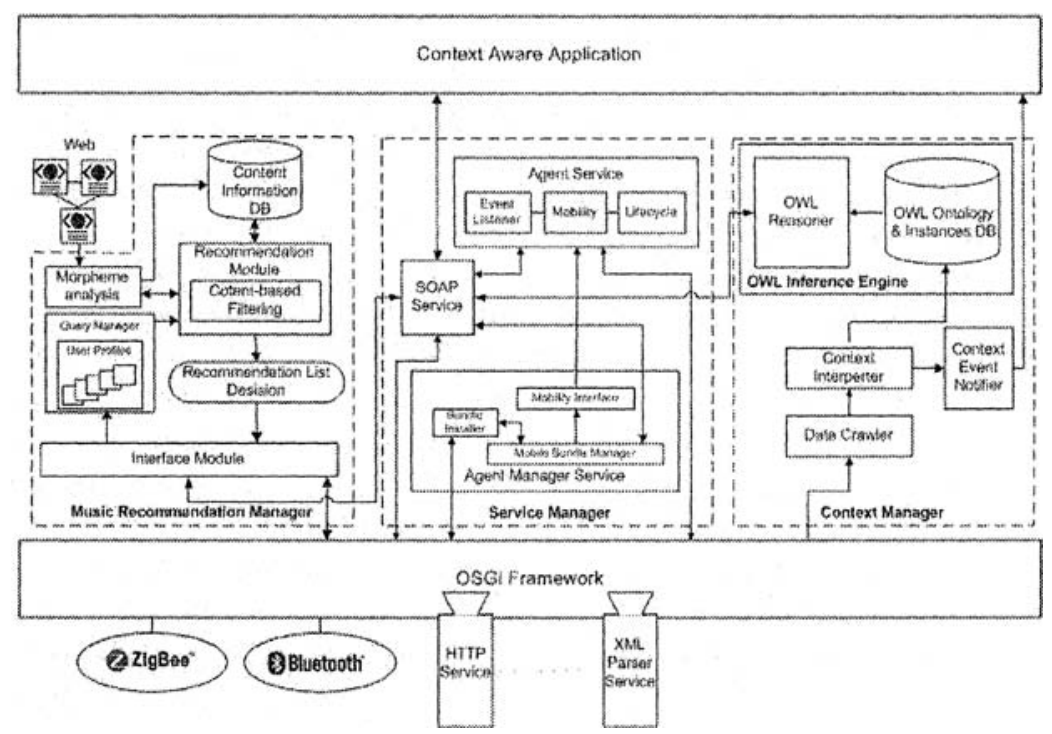

Fig. 2. Ontology for Context in Content-based Music Recommendation System

The Context Manager transfered data generated by events (sensing) to a context analyzer and that data was transfered to an OWL inference engine. The OWL inference engine transferred data received from the context manager to the Service Manager in which data was transformed as information using an OWL inferencer including OWL ontology object DB. 
The Inference Engine in the Context Manager used an ontology inferencer Jena 2.0 .

The Service Manager consisted of a Bundle Service that provided music recommendation service as a bundle in a Simple Object Access Protocol (SOAP) Service, OSGi framework installed device in order to transfer information received from the OWL inference engine to the music recommendation system, and an Application and Bundle Manager Service that supported the management of the mobility of bundles. Communication between the Context Manager, Music Recommendation Manager, and Music Service can be performed using the SOAP Service. This service makes possible a real-time process in music recommendation service using the context information between different systems. The Bundle Manager Service automatically manages Bundles and supports service mobility. Service mobility in a music recommendation system means that service is not interrupted by a different device that has an OSGi middleware even though a user's location has changed.

The Music Recommendation Manager played a role in the decision of an optimal music recommendation list in a recommendation module by applying the recommendation list that corresponded to certain context information received from the Service Manager with the user profile and MCIDB to a filtering process in a recommendation module. The Query Manager analyzed the context information received from the Service Manager, selected a proper profile according to user context information, and transfered it to the Recommendation Module. In addition, it performed an updating process for the music selected by users in the User Profile. The Recommendation Module performed the Morpheme analysis of the profile received from the Query Manager and configured a recommendation list by searching music from the MCIDB. The configured music list was ranked by the Recommendation List Decision and that was transferred as a new list to the Interface Module. The new list was then transferred to the Application through the SOAP Service.

\section{EXPERIMENT}

This chapter tested whether user and context information is correctly recognized in an OSGi framework and whether a proper recommendation music list was provided by transferring this information to the Music Recommendation Manager in order to verify the CBMRS proposed in this study. The test environment consisted of the Context Manager and Service Manager as a bundle in an OSGi gateway on a home network in which the 
Music Recommendation Manager was implemented on a desktop Personal Computer (PC). In addition, the MCIDB consisted of 300 pop songs.

Fig. 3 presents the output of context information produced by the Context Manager. The output was able to recognize the information as that of user Jong-Hun: age is Adult, sex is male, pulsation is normal, weather is snow, temperature is cold, and location is Guestroom. The transferred context information was used to make a proper music recommendation list for user context through the Music Recommendation Manager and was output to the $\mathrm{PC}$ in the Guestroom.

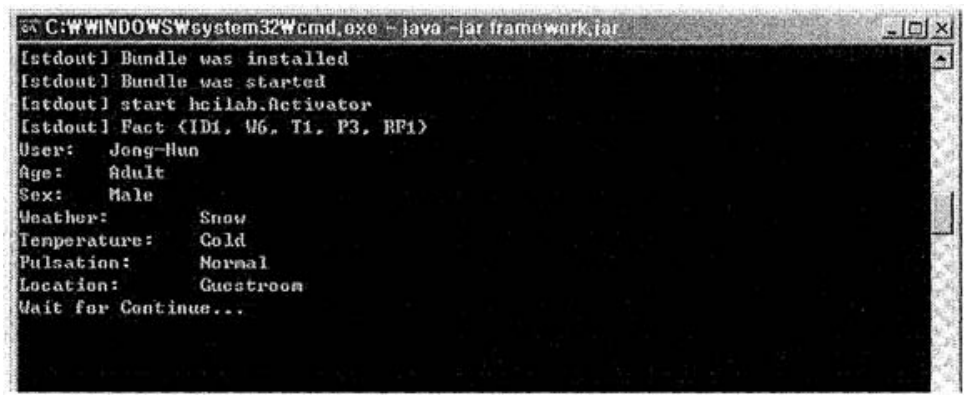

Fig. 3. An Execution View of Context Mạnager

In the paper, survey is used to evaluation of system. 50 users participated in system estimation. Users responded by value to $1-5$ on question of table 3. Table 3 showed that user's satisfaction for system is high on the whole.

Table 3. Questionnaire and Results

\begin{tabular}{|l|c|}
\hline \multicolumn{1}{|c|}{ Questionnaire } & Result (average of users' value sum $* 20)$ \\
\hline Does the weather influence in music selection? & $79.2 \%$ \\
\hline Is music that recommend satisfied? & $93.2 \%$ \\
\hline Do you think that CBMRS is useful? & $94.4 \%$ \\
\hline
\end{tabular}

\section{CONCLUSIONS AND FUTURE WORK}

A music search and recommendation system that uses the conventional music search and recommendation method performs music search and recommendation by analyzing certain information that is passively received from users. This method presents a difficulty in a proper searching result for users' circumstances. Also, it has some problems that have to receive a lot of expressions from users in order to increase the satisfaction of users. 
This paper actively obtained and recognized user context information and designed a Content-based Music Recommendation System (CBMRS) that is appropriate to the user using the recommendation and inquiry of the contentbased music recommendation system. The context introduced in this study was defined as ontology in order to use it as context information. In addition, a content-based filtering method, which was based on a statistical method, was developed. Also, this system was designed based on an OSGi framework in order to provide services without any interruptions wherever and whenever in a home network and that increased user satisfaction.

As a future study, a new filtering method that considers user information to solve a specialization trend, which doesn't exceed the limited preferences of the CBMRS, is required. Finally, a middleware that is able to perform music recommendations and services without any interruptions to other services by considering a users various context information in a home network, is also necessary.

\section{ACKNOWLEDGEMENT}

This research was supported by the Brain Korea 21 Project in 2006.

\section{REFERENCES}

1. H. -C. Chen, and A. L. P. Chen, "A music recommendation system based on music data grouping and user interests," Proc. of the CIKM'01 (2001) 231-238

2. P. J. Brown, I. D. Bovey, and X. Chen, "Context-Aware Application: From the Laboratory to the Marketplace," IEEE Personal Communication (1997) 58-64

3. M. Balabanovic, and Y. Shoham, "Fab; Content-based, Collaborative Recommendation," Communication of the Association of Computing Machinery, Vol. 40, No, 3 (1997) 6672

4. K. Y. Jung, and J. H. Lee, "User Preference Mining through Hybrid Collaborative Filtering and Content-based Filtering in Recommendation System," IElCE Transaction on Information and Systems, Vol, E87-D, No. 12 (2004) 2781-2790

5. P. Dobrev, D. Famolari, C. Kurzke, and B. A. Miller, "Device and Service Discovery in Home Networks with OSGi," IEEE Communications Magazine, Vol. 40, Issue 8, August (2002) $86-92$

6. C. Basu, H. Hirsh, and W. W. Cohen, "Recommendation as classification; Using social and content-based information in recommendation," In proceedings of the Fitteenth National Conference on Artificial Intelligence (1998) 714-720

7. K. Romer, T. Schoch, F, Mattern, and T. Dubendorfer, "Smart Identification Frameworks for Ubiquitous Computing Application," IEEE International Conference on Pervasive Computing and Communication (2003)

8. T. Gu, H. K. Pung, and D. Q. Zhang, "An Ontology-based Context Model in Intelligent Environments," Proceedings of Communication Networks and Distributed Systems Modeling and Simulation Conference (2004) 270-275 